\title{
Antikoagulation bei Herzinsuffizienz
}

\author{
Gregor Simonis
}

\begin{tabular}{|c|c|c|c|}
\hline \multicolumn{4}{|l|}{ Übersicht } \\
\hline Einleitung & 47 & Müssen Patienten mit & \\
\hline Herzinsuffizienz mit Vorhof- & & eingeschränkter linksventrikulärer & \\
\hline flimmern - was ist besonders & & Pumpfunktion und Sinusrhythmus & \\
\hline zu beachten? & 48 & antikoaguliert werden? & 51 \\
\hline Vorhofflattern und atriale & & Ist die Gabe von ASS sinnvoll? & 53 \\
\hline Tachykardien & 49 & Antikoagulation bei intrakardialen & \\
\hline „Valvuläre“ Herzinsuffizienz & 50 & Thromben & 53 \\
\hline & & Sonderfall: Non-Compaction- & \\
\hline & & Kardiomyopathie & 55 \\
\hline & & Was sagen die Leitlinien & 55 \\
\hline
\end{tabular}

\section{Einleitung}

Herzinsuffizienz ist vereinfacht definiert die Unfähigkeit des Herzens, die Körperperipherie mit einem ausreichenden Herzzeitvolumen zu versorgen. Dieses erniedrigte Herzzeitvolumen impliziert nach klassischer Betrachtungsweise eine Veränderung der Virchow-Trias, die Veränderungen des Blutflusses, der Endothelbeschaffenheit und der Blutzusammensetzung zusammenfasst und als prädisponierend für thrombotische Erkrankungen beschreibt. Typische Veränderungen der Thrombogenese bei Herzinsuffizienz sind in Abb. 1 summiert.

Auf der Basis von 3 in den 1950er Jahren durchgeführten randomisierten Studien - mit deutlicher Reduktion der Mortalität herzinsuffizienter Patienten (Übersicht bei [2]) - antikoagulieren viele ältere Kliniker ihre Patienten mit schwerer Herzinsuffizienz. Es muss aber angemerkt werden, dass die in diesen Studien untersuchten Patienten überwiegend an postrheumatischer, valvulärer Herzinsuffizienz (s.u.) erkrankt waren und dass die Studiendesigns nicht so stringent waren, wie das heute üblich ist.

Das aktuelle Verständnis der Herzinsuffizienz ist das eines Symptomenkomplexes unterschiedlicher Ätiologie. Hierbei wird die Diagnose Herzinsuffizienz traditionell klinisch als kardial bedingte Luftnot und Belastungsinsuffizienz gestellt, woraus sich ein ätiologisches
Spektrum von symptomatischem Vorhofflimmern (das bei einer entsprechenden diastolischen Dysfunktion der Herzinsuffizienz mit erhaltener LV-Funktion zuzuordnen sein kann) über intrakardiale Raumforderungen bis hin zu Erkrankungen mit höhergradiger Einschränkung der linksventrikulären Pumpfunktion ergibt. Die Frage, ob bzw. bei welchen Formen der Herzinsuffizienz eine Antikoagulation sinnvoll ist, bleibt trotzdem aktuell und wurde in den vergangenen Jahren durch eine Reihe randomisierter Studien unter-

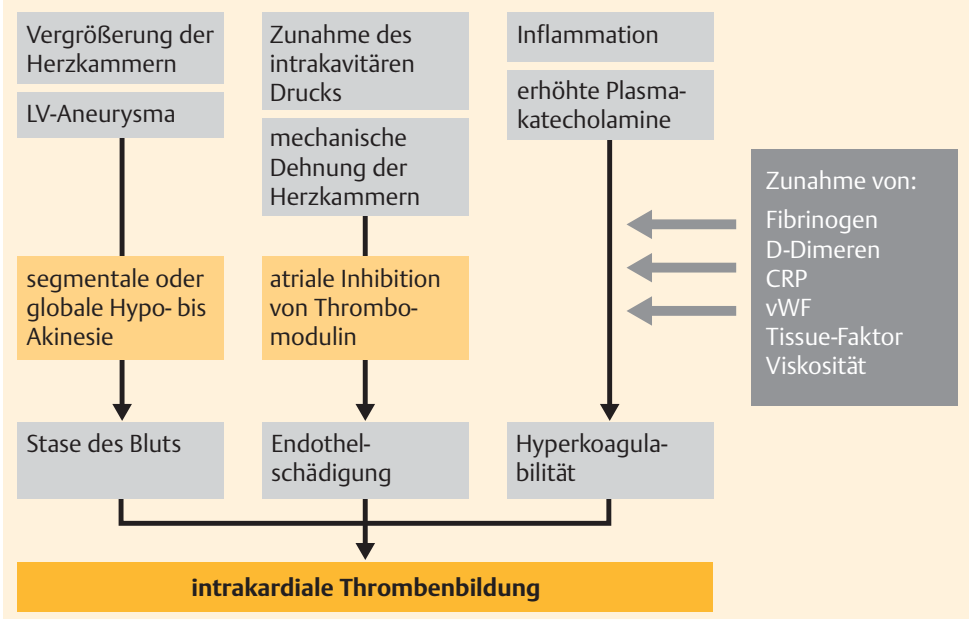

Abb. 1 Antikoagulation und Thrombozytenaggregation bei Herzinsuffizienz: Pathophysiologie der kardialen Thrombogenese; $C R P=C$-reaktives Protein, vWF = Von-WillebrandFaktor (modifiziert nach [1]). 
sucht. In vielen Situationen wurde vor allem der Stellenwert der neuen, direkten oralen Antikoagulanzien (NOAKs) hinterfragt. Der vorliegende Artikel soll die vorliegende Evidenz für unterschiedliche Formen der Herzinsuffizienz zusammenfassen und kritisch bewerten.

\section{Herzinsuffizienz mit Vorhofflimmern - was ist besonders zu beachten?}

\section{Indikation nach Risikoprofil}

Bei Vorhofflimmern wird die Antikoagulationsindikation grundsätzlich nach dem Risikoprofil gestellt, das nach neuesten Leitlinien mit dem $\mathrm{CHA}_{2} \mathrm{DS}_{2}$ VASc-Score abgeschätzt wird [3,4]. Die Herzinsuffizienz-in der Originalpublikation definiert als in letzter Zeit behandlungswürdige Symptomatik [5] - , in den aktuellen Leitlinien prononciert auf die systolische Herzinsuffizienz mit $\mathrm{EF}<40 \%$, ist obligat schon ein Risikopunkt im genannten Score. Da bei Herzinsuffizienzpatienten fast regelhaft Begleiterkrankungen wie Diabetes, Hypertonie, KHK oder ein fortgeschrittenes Lebensalter vorliegen, ist bei nahezu allen Herzinsuffizienzpatienten mit Vorhofflimmern eine dauerhafte Antikoagulation indiziert.

\section{Typ des Vorhofflimmerns}

Für die Indikation zur Antikoagulation ist es unerheblich, ob die Patienten paroxysmales, persistierendes oder permanentes Vorhofflimmern haben. Dies konnten Hohnloser und Mitarbeiter in einer Subanalyse der ACTIVE-W-Studie zeigen [6]: Obwohl die 1202 Patienten mit paroxysmalem Vorhofflimmern in dieser Analyse jünger waren und eine geringere Prävalenz an Herzinsuffizienz oder Diabetes aufwiesen, hatten sie mit 2,0\%/Jahr eine vergleichbare Thromboembolierate wie die 5495 Patienten mit permantentem Vorhofflimmern (2,2\%/Jahr). Die plasmatische Antikoagulation mit Kumarinen war in beiden Kollektiven mit einer vergleichbaren Ereignisreduktion verbunden. Somit besteht bei jedem Patienten mit Herzinsuffizienz, der anamnestisch bereits eine oder mehrere stattgehabte Vorhofflimmerepisoden aufweist und sich aktuell im Sinusrhythmus präsentiert, eine Indikation zur oralen Antikoagulation.
Alle Patienten mit Vorhofflimmern und erhöhtem $\mathrm{CHA}_{2} \mathrm{DS}_{2}$ VASc-Score, z. B. bei Herzinsuffizienz, werden antikoaguliert- unabhängig vom Typ des Vorhofflimmerns.

\section{Therapie mit NOAKs}

Die Frage, ob NOAKs eine sinnvolle Therapie bei Vorhofflimmern sind, wurde in den großen Studien RE-LY [7], Rocket-AF [8] und Aristotle [9] überprüft. Die genannten Studien haben grundsätzlich Vorhofflimmerpatienten mit Thromboembolierisiko eingeschlossen, was im Sinne des in den Studien genutzten $\mathrm{CHADS}_{2}$-Scores zwangsläufig auch zum Einschluss herzinsuffizienter Patienten geführt hat. Grundergebnis aller Studien war, dass NOAKs im Vergleich zu Kumarinen effektiver sind und/oder die mit ihnen einhergehende Blutungswahrscheinlichkeit geringer ist, sodass die aktuellen Leitlinien zur Therapie von Patienten mit Vorhofflimmern NOAKs aufgrund ihres Nettobenefits Kumarinen vorziehen (Empfehlungsgrad IIb, Evidenzklasse A) [4].

Für die Frage, ob NOAKs bei Patienten mit Herzinsuffizienz sinnvoll sind, sind schlussendlich aber nicht die Gesamtresultate der Studien entscheidend, sondern die Daten zur Schlaganfallprävention und die Daten zu Blutungsereignissen in der Subgruppe der Patienten mit Herzinsuffizienz. Insbesondere nach warnenden Publikationen hinsichtlich vermehrter Blutungen bei kritischer kranken Patienten [10] und dem daraus resultierenden, im Jahr 2011 verschickten Rote-HandBrief zu Pradaxa [11] erscheint es kritisch, diesen Punkt zu hinterfragen. Die Daten sind in der Regel nur in Online-Supplements oder Substudien der großen Arbeiten verfügbar (Abb.2, Abb.3). Insgesamt zeigt sich sowohl hinsichtlich des Wirksamkeitsendpunkts (Schlaganfälle/periphere Embolien) als auch des Sicherheitsendpunkts (Blutungen) ein mit dem Gesamtkollektiv vergleichbares Ergebnis ohne Hinweis auf Sicherheitsprobleme. Es sei aber darauf hingewiesen, dass eine Dekompensation einer Herzinsuffizienz fast regelhaft mit einer Verschlechterung der Nierenfunktion einhergeht. Sollten Patienten mit einer solchen Dekompensation Dabigatran erhalten, müssen die Nierenwerte engmaschig kontrolliert, die Dosis ggf. angepasst und das Medikament bei einer GFR $<30 \mathrm{ml} /$ min abgesetzt werden, weil Dabigatran überwiegend renal eliminiert wird und daher bei schlechter Nierenfunktion kumulieren kann. Da es hierzu 2011 den erwähnten Rote-Hand-Brief gegeben hat [11], erscheint die Beachtung dieser Maßnahmen auch medizinrecht- 


\begin{tabular}{|c|c|c|c|c|c|c|c|c|}
\hline Subgruppe & Patienten & \multicolumn{3}{|c|}{ Dabigatran } & $\begin{array}{c}\text { Hazard-Ratio mit } \\
\text { Dabigatran } 110 \mathrm{mg} \\
(95 \% \mathrm{Cl})\end{array}$ & $\underset{\text { (Interaktion) }}{\mathrm{p}}$ & $\begin{array}{l}\text { Hazard-Ratio mit } \\
\text { Dabigatran } 150 \mathrm{mg} \\
(95 \% \mathrm{Cl})\end{array}$ & $\stackrel{p}{p}$ \\
\hline symptomatis & ienz & \multicolumn{2}{|c|}{ \% pro Jahr } & & & \multirow[t]{2}{*}{0,42} & & 0,33 \\
\hline ja & 4904 & 1,90 & 1,44 & 1,85 & - & & : & \\
\hline nein & 13203 & 1,40 & 1,00 & 1,63 & & & & \\
\hline
\end{tabular}

Rivaroxaban: Rocket AF

\begin{tabular}{|c|c|c|c|c|c|c|}
\hline & \multicolumn{6}{|c|}{ Ereignisse } \\
\hline & & & Rivaroxaban & Warfarin & $\mathrm{p}$-Wert & p (Interaktion) \\
\hline \multirow[t]{3}{*}{ Herzinsuffizienz } & ja & -1 & $106 / 4428(2,39 \%)$ & $141 / 4409(3,2 \%)$ & $0,76(0,59,0,98)$ & 0,664 \\
\hline & nein & $\rightarrow-$ & $83 / 2632(3,15 \%)$ & $102 / 2672(3,82 \%)$ & $0,83(0,62,1,11)$ & \\
\hline & Rivarox & 1 & $\begin{array}{l}0 \\
\text { n schlechter }\end{array}$ & & & \\
\hline
\end{tabular}

\section{Apixaban: Aristotle}

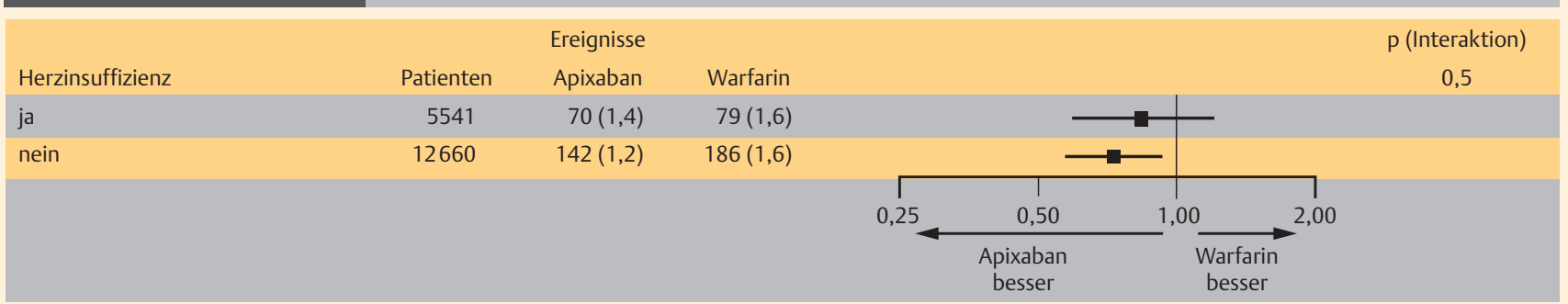

Abb. 2 Schlaganfälle oder systemische Embolien bei Patienten mit und ohne Herzinsuffizienz in den Studien zu neuen Antikoagulanzien.

lich relevant. Die beiden aktuell verfügbaren oralen Faktor-Xa-Hemmer Rivaroxaban und Apixaban sind bis zu einer GFR von $15 \mathrm{ml} / \mathrm{min}$ zugelassen und sollten, sofern nicht parallel eine relevante Leberstoffwechselstörung vorliegt (der Abbau dieser Medikamente verläuft überwiegend hepatisch, daher sind sie bei Leberzirrhose in den Stadien Child-Pugh B und C kontraindiziert), unkomplizierter zu handhaben sein.

Patienten mit Vorhofflimmern und Herzinsuffizienz können mit NOAKs behandelt werden. Regelmäßige Nierenwertkontrollen sind vor allem bei Dekompensationen obligat.

\section{Vorhofflattern und atriale Tachykardien}

\section{Indikation nach Risikoprofil}

Die Antikoagulation von Patienten mit Vorhofflattern und atrialen Tachykardien orientiert sich grundsätzlich an der Behandlung des Vorhofflimmerns. Da Patienten mit Herzinsuffizienz per se einen erhöhten
$\mathrm{CHA}_{2} \mathrm{DS}_{2}$ VASc-Score aufweisen, ist hier in der Regel auch eine dauerhafte Antikoagulation obligat.

\section{Antikoagulation nach Ablation}

Vorhofflattern und atriale Tachykardien, deren medikamentöse Frequenzregulierung erfahrungsgemäß schlechter gelingt als die des Vorhofflimmerns, werden vermehrt mittels Katheterablation behandelt. Die Frage, ob nach erfolgreicher Ablationsbehandlung weiter antikoaguliert werden muss, wird kontrovers diskutiert. Nach Ablation eines isoliert rechtsatrialen, typischen Vorhofflatterns und längerfristiger Rezidivfreiheit kann eventuell diskutiert werden, ob die Antikoagulation beendet wird, weil das Substrat der Rhythmusstörung eindeutig therapiert wurde. Bei linksatrialen Rhythmusstörungen (atypisches Vorhofflattern, atriale Tachykardien) und/oder hohem $\mathrm{CHA}_{2} \mathrm{DS}_{2}$ VAScScore erscheint dagegen die weitere Antikoagulation Vorgehen der Wahl zu sein, weil der erkrankte Vorhof in der Regel als Substrat der Rhythmusstörung persistiert und die Rezidivrate nach Ablation höher ist als bei typischem Vorhofflattern. Hierzu gibt es aber keine klaren Empfehlungen in Leitlinien. 


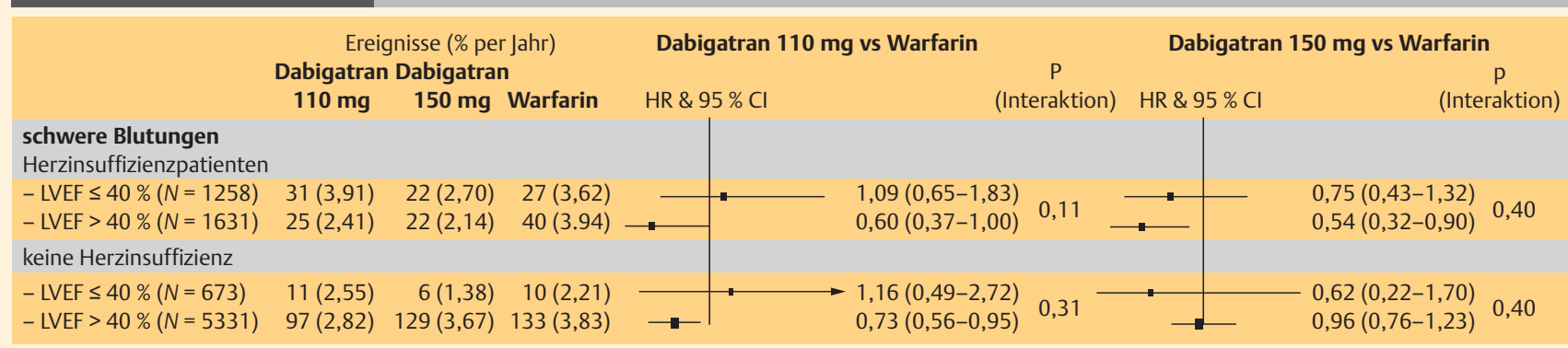

\section{Rivaroxaban}

\begin{tabular}{|c|c|c|c|c|}
\hline \multirow[t]{2}{*}{ schwere oder klinisch relevante Blutungen } & \multicolumn{3}{|r|}{15} & \multirow{2}{*}{$\begin{array}{c}\mathrm{p} \\
\text { (Interaktion) }\end{array}$} \\
\hline & Rivaroxaban & Warfarin & HR (95\% Cl) & \\
\hline alle Patienten & $1475(20,74)$ & $1449(20,34)$ & $1,03(0,96,1,11)$ & \\
\hline Patienten mit Herzinsuffizienz & $864(19,39)$ & $859(19,36)$ & $1,01(0,92,1,11)$ & 0,587 \\
\hline
\end{tabular}

\section{Apixaban}

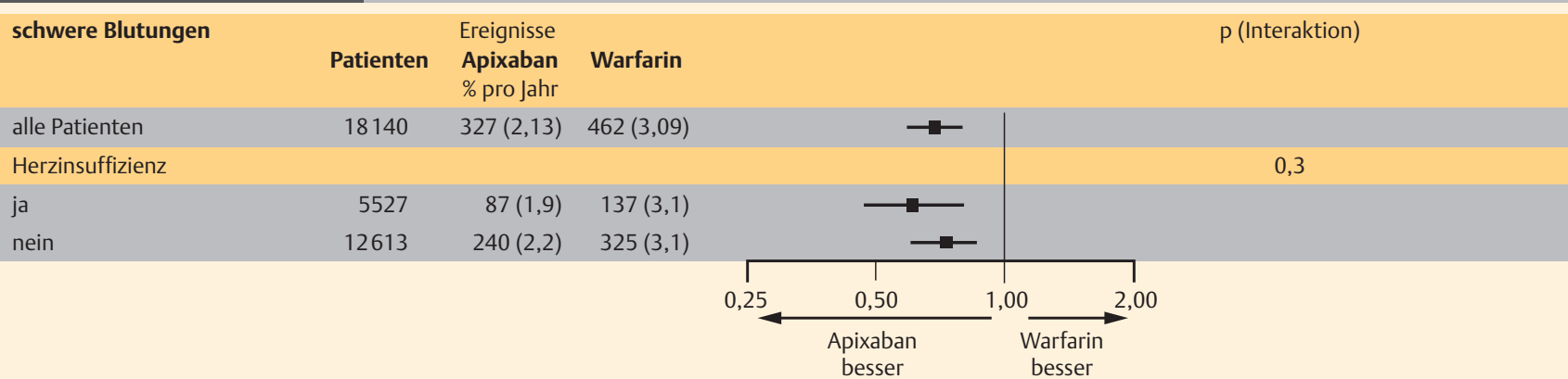

Abb. 3 Blutungsereignisse bei Patienten mit und ohne Herzinsuffizienz in den Studien zu neuen Antikoagulanzien.

\section{„Valvuläre“ Herzinsuffizienz}

Im Zusammenhang mit der gesteigerten Aufmerksamkeit gegenüber der Antikoagulation bei Vorhofflimmern wird die Frage, was „valvuläres“ Vorhofflimmern und in Konsequenz möglicherweise „valvuläre“ Herzinsuffizienz ist, aktuell immer wieder diskutiert. Hintergrund ist, dass die NOAKs nur für „non-valvuläres“ Vorhofflimmern getestet und zugelassen sind, eine Nutzung bei „valvulären“ Erkrankungen also „off-label“ und mindestens aufklärungspflichtig ist. Daher soll an dieser Stelle auf 2 Sonderfälle hingewiesen werden:

- Oft wird vergessen, dass der Terminus „valvuläres Vorhofflimmern" historisch gesehen die postrheumatischen Vitien beschreibt. Dabei handelt es sich in der Regel um Mitralstenosen und seltener Aortenklappenstenosen bei Patienten meist mittleren Alters - und hinsichtlich ihrer Thromboemboliegefahr um eine eigene Entität: Gemäß epidemiologischen
Daten aus der Framingham-Studie (1982) ist die Thromboemboliegefahr bei alleinigem Vorhofflimmern 5-mal höher als beim Sinusrhythmus, bei Vorhofflimmern und Z.n. rheumatischem Fieber aber sogar 17-mal höher [12]. In Konsequenz werden Patienten mit höhergradigen Mitralvitien ab einem Durchmesser des linken Vorhofs von $50 \mathrm{~mm}$ unabhängig vom aktuellen Vorhofrhythmus immer antikoaguliert. Für diese Patienten ist die Risikoeinschätzung mittels des $\mathrm{CHADS}_{2}$-Scores obsolet. Die Nutzung neuer Antikoagulanzien ist bei diesen in Westeuropa sehr seltenen Patienten nicht evidenzbasiert.

- Bei Patienten mit mechanischen Herzklappen führt Dabigatran auch in hohen Dosierungen bis $600 \mathrm{mg} / \mathrm{d}$ im Vergleich zu Kumarinen zu mehr thromboembolischen Ereignissen und mehr Blutungen [13]. Daher ist Dabigatran bei mechanischen Herzklappen kontraindiziert. Die Gabe anderer NOAKs ist ohne vorliegende Daten definitiv nicht empfehlenswert. Warum Dabi- 
gatran bei mechanischen Herzklappen nicht so effektiv ist wie Warfarin, ist nicht völlig geklärt. Die Autoren der Studie vermuten, dass die Gerinnungsaktivierung bei mechanischen Herzklappen mehr über den „tissue factor“ und den direkten Kontakt der künstlichen Oberflächen mit dem Blut geschieht, was beides durch den direkten Thrombininhibitor weniger gehemmt wird als durch die an vielen Gerinnungsfaktoren angreifenden Kumarine, die die genannten Gerinnungswege durch Inhibition der Faktor-VII- und Faktor-XI-Bildung direkt adressieren.

Im Gegensatz dazu bedürfen biologische Herzklappen keiner Antikoagulation, sondern können mit ASS geführt werden. Ist bei Patienten mit biologischen Herzklappen aufgrund anderer Erkrankungen eine Antikoagulation indiziert, können NOAKs daher grundsätzlich gegeben werden.

Eine valvuläre Herzinsuffizienz im Sinne einer Kontraindikation zur NOAK-Gabe ist nur bei postrheumatischen Vitien und prothetischen Herzklappen gegeben.

Dieser Standpunkt wird von den aktuellen Vorhofflimmerleitlinien so geteilt (Kap.4.1.1 der Leitlinien) [3]. Bei allen anderen Vitien, wie z.B. höhergradigen sekundären AV-Klappen-Insuffizienzen, sind NOAKs nicht kontraindiziert.

\section{Müssen Patienten mit ein- geschränkter linksventrikulärer Pumpfunktion und Sinusrhyth- mus antikoaguliert werden?}

\footnotetext{
Die Herzinsuffizienz mit eingeschränkter LV-Funktion geht, wie eingangs beschrieben, fast regelhaft mit einem reduzierten Herzzeitvolumen einher. Darüber hinaus beschreiben einige Autoren auch eine Hyperkoagulabilität bei diesen Patienten mit der resultierenden Bildung linksventrikulärer Thromben und einer erhöhten Schlaganfallrate [14]. Autopsiestudien weisen darauf hin, dass die Rate atherothrombotischer Ereignisse bei den Patienten mit Herzinsuffizienz erhöht ist, die am grundsätzlich primär rhythmogen attributierten plötzlichen Herztod verstorben sind [15]. Zum anderen haben viele Patienten mit eingeschränkter LVFunktion unbemerkte Vorhofflimmerepisoden, die ein zusätzliches Thromboembolierisiko beinhalten. Diese Daten lassen die Annahme, dass Patienten mit einge-
}

schränkter LV-Funktion auch im Sinusrhythmus von einer Antikoagulation profitieren, intuitiv richtig erscheinen.

Zur Klärung dieser Fragestellung wurden 4 randomisierte Studien durchgeführt:

- HELAS-Studie: In diese Studie wurden 197 Patienten mit einer LV-EF $\leq 35 \%$ eingeschlossen und doppelblind in eine plasmatische Antikoagulation oder ASS $325 \mathrm{mg} / \mathrm{d}$ (bei ischämischer Kardiomyopathie) bzw. Placebo (bei dilatativer Kardiomyopathie) randomisiert [16]. Primärer Endpunkt war die Kombination aus Schlaganfall, Myokardinfarkt, peripherer Embolie, Lungenembolie, Hospitalisation aus kardialer Ursache und Tod jeglicher Ursache. Die Patienten wurden im Mittel 22 Monate nachverfolgt. Die Häufigkeit embolischer Ereignisse unterschied sich nicht und betrug im Mittel 2,2 pro 100 Patientenjahre, während nicht mit embolischen Ereignissen assoziierte Endpunkte wie Hospitalisationen wegen Herzinsuffizienz mit 15 pro 100 Patientenjahren unter ASS deutlich häufiger auftraten. In der antikoagulierten Gruppe traten immerhin 4,9 relevante Blutungen pro 100 Patientenjahre auf-im Vergleich zu keinen Blutungen in der Kontrollgruppe.

- WASH-Studie: Die offen geführte WASH-Studie randomisierte 279 Patienten mit symptomatischer systolischer Herzinsuffizienz in keine Therapie, ASS oder Kumarintherapie [17]. Die Randomisierung berücksichtigte nicht, ob eine KHK vorlag oder nicht, d.h. in der Gruppe ohne Therapie waren ebenfalls ca. $60 \%$ KHK-Patienten, die formal eine Indikation zur Thrombozytenaggregationshemmung hatten. Der primäre Endpunkt war die Kombination aus Tod, Schlaganfall oder Myokardinfarkt und wurde während der mittleren Nachverfolgungszeit von 27 Monaten von 26\%, 32\% und 26\% der jeweiligen Patientengruppe erreicht, was aufgrund der relativ geringen Patientenzahl nicht signifikant war. Die sehr hohe Ereignisrate war getrieben durch die hohe Zahl von Todesfällen im Nachverfolgungszeitraum (19\%, $25 \%, 22 \%$ ), die ätiologisch nicht weiter aufgeschlüsselt wurde. Es bleibt also unklar, ob die Todesfälle eine Relation zu Thromboembolien hatten. Die eindeutig „gerinnungsassoziierten“ Ereignisse Schlaganfall und Myokardinfarkt weisen andererseits auf einen Vorteil für die Therapiegruppen hin (7\%, 4\%, $1 \%$ ) mit der Limitation der geringen Ereignisrate und entsprechend niedriger Aussagekraft. Auch in dieser Studie waren Hospitalisationen wegen Herzinsuffizienz deutlich häufiger als thromboembolische Ereignisse und kamen in der ASS-Gruppe öfter vor als in den anderen beiden Gruppen (19\%, 34\%, 20\%). Die 


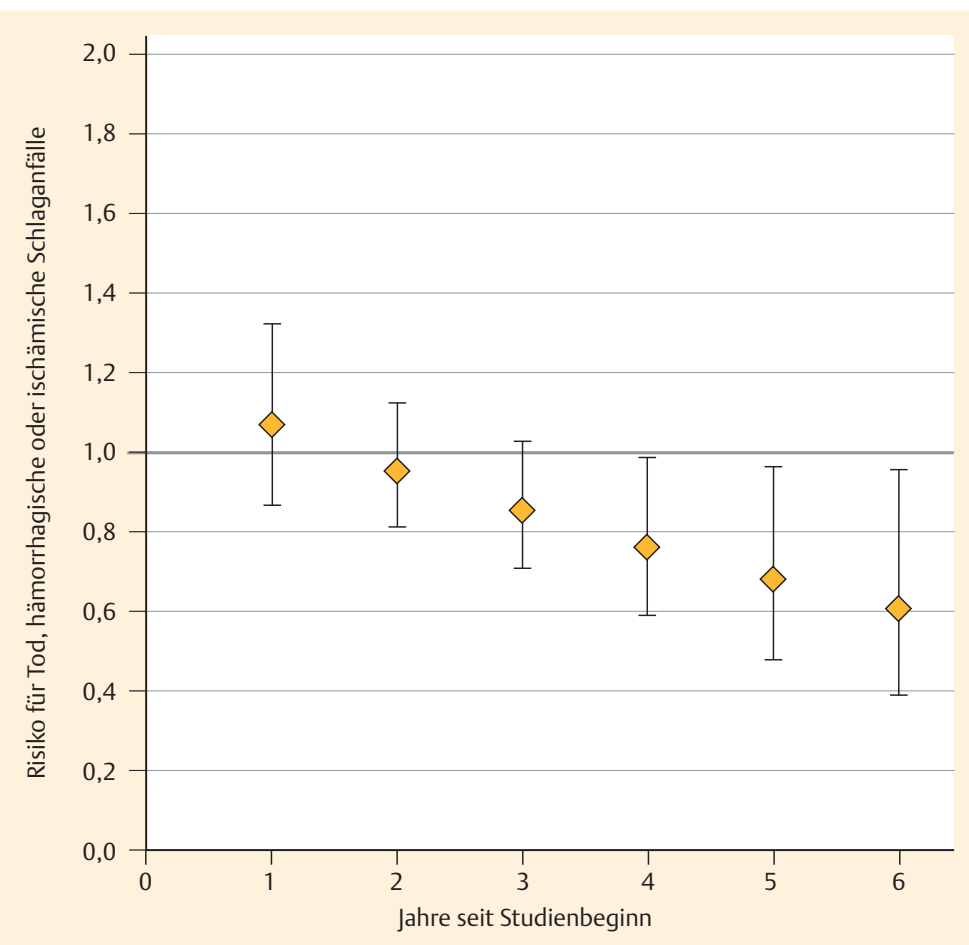

Abb. 4 Risiko für Tod, hämorrhagische oder embolische Schlaganfälle unter plasmatischer Antikoagulation im Vergleich zur ASS-Therapie: Subanalyse aus der WARCEF-Studie [19]).

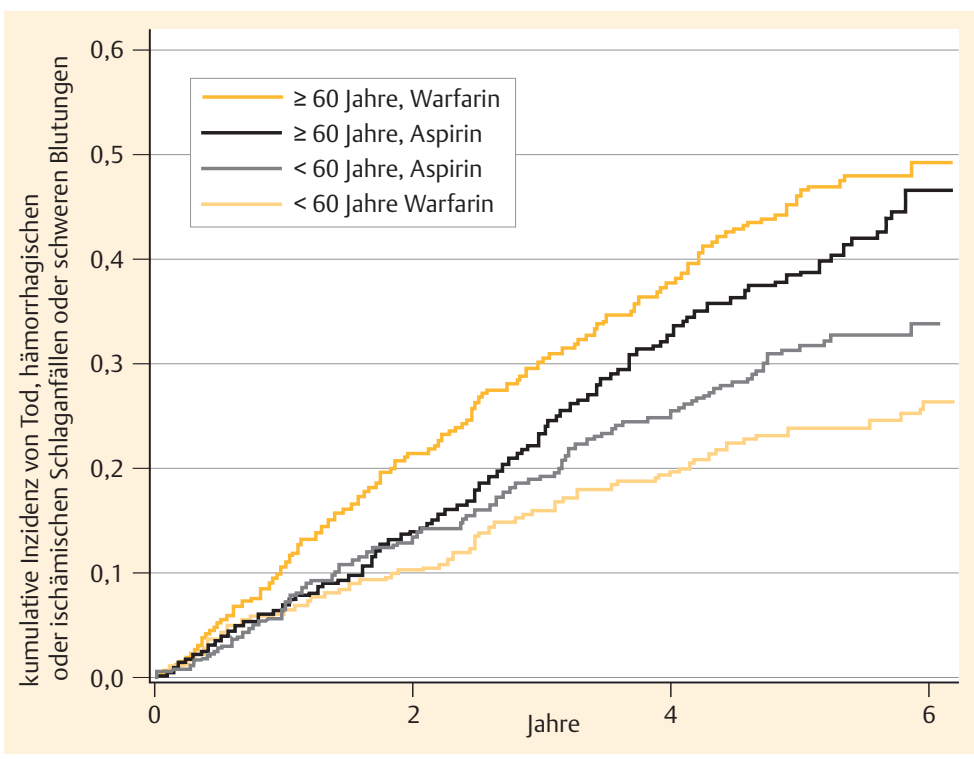

Abb.5 Jüngere Patienten haben unter Warfarin weniger Ereignisse als unter Azetylsalizylsäure: Subanalyse aus der WARCEF-Studie [20].

Zahl der schweren Blutungen war gering ( $0 \%, 1 \%$, $4 \%)$. Die Autoren folgerten, dass zumindest die ASSTherapie keine eindeutigen Stellenwert in der Herzinsuffizienztherapie hat.

- WATCH-Studie: Die deutlich größere WATCH-Studie [18] randomisierte 1587 Patienten mit symptomati- scher, stabiler Herzinsuffizienz, einer $\mathrm{LV}-\mathrm{EF} \leq 35 \%$ und Sinusrhythmus in eine plasmatische Antikoagulation (INR 2,5 - 3), Azetylsalizylsäure oder Clopidogrel. Primärer Endpunkt war wie in der WASH-Studie die Kombination von Tod, Myokardinfarkt und Schlaganfall mit einem mittleren Nachverfolgungsintervall von 1,9 Jahren. Auch in dieser Studie unterschied sich die Häufigkeit des primären Endpunkts nicht (Warfarin: 19,6\%, Azetylsalizylsäure: 20,7\%, Clopidogrel: $21,6 \%$ ), die Kombination von Myokardinfarkten und Schlaganfällen $(4,0 \%, 4,1 \%, 4,4 \%)$ ergab keine Unterschiede und die Hospitalisationsrate wegen dekompensierter Herzinsuffizienz war ebenfalls hoch und unter ASS (22\%) höher als in den anderen beiden Studienarmen (Warfarin 16\%, Clopidogrel 18\%). Hingegen waren die Blutungsraten unter Warfarin (5,4\%) höher als unter ASS oder Clopidogrel (3,6\%, 2,1\%).

- WARCEF-Studie: WARCEF [19] war die mit 2305 randomisierten Patienten größte Studie mit der längsten mittleren Nachverfolgung der Patienten (3,05 Jahre). In WARCEF erhielten Patienten mit Sinusrhythmus und eingeschränkter LV-Funktion entweder Warfarin (INR 2,0 - 3,5) oder ASS (325 mg/d). Der primäre Endpunkt war die Kombination aus ischämischem oder hämorrhagischem Schlaganfall und der Gesamtmortalität. Dieser Endpunkt war mit 7,47 vs. 7,93/100 Patientenjahre nicht signifikant unterschiedlich. Die unter Warfarin gesehene Reduktion ischämischer Schlaganfälle ( $0,72 \%$ vs. $1,36 \%)$, die auch in den anderen Studien im Trend nachweisbar war, wurde durch eine erhöhte Hämorrhagierate kompensiert. Ein wirklich interessantes und hypothesengenerierendes Ergebnis der Studie war, dass die Ereignisrate in den ersten Behandlungsjahren unter Warfarin höher war als unter ASS, sich aber längerfristig dieses Verhältnis umgekehrt hat (Abb.4). Schlussfolgerung der Studienautoren war, dass die antithrombotische Therapie idealerweise zu individualisieren ist. In einer sehr lesenswerten Subgruppenanalyse der WARCEF-Studie [20] wurde versucht, klinische Prädiktoren für einen vermehrten Benefit von einer Warfarin- oder ASS-Therapie zu differenzieren. Hier zeigt sich sehr konsistent, dass die plasmatische Antikoagulation vor allem jüngeren Patienten $(<60$ Jahre) nutzt, während sie bei älteren Patienten keinen Vorteil gegenüber einer ASS-Therapie bringt. Dieses Ergebnis ist auch dann konsistent, wenn schwere Blutungen zum primären Endpunkt Mortalität und Schlaganfälle addiert werden (Abb.5).

Die 4 genannten Studien wurden in 2 Metaanalysen unter Verwendung der individuellen Datensätze eingebracht $[21,22]$. Hier zeigte sich, dass Warfarin die 


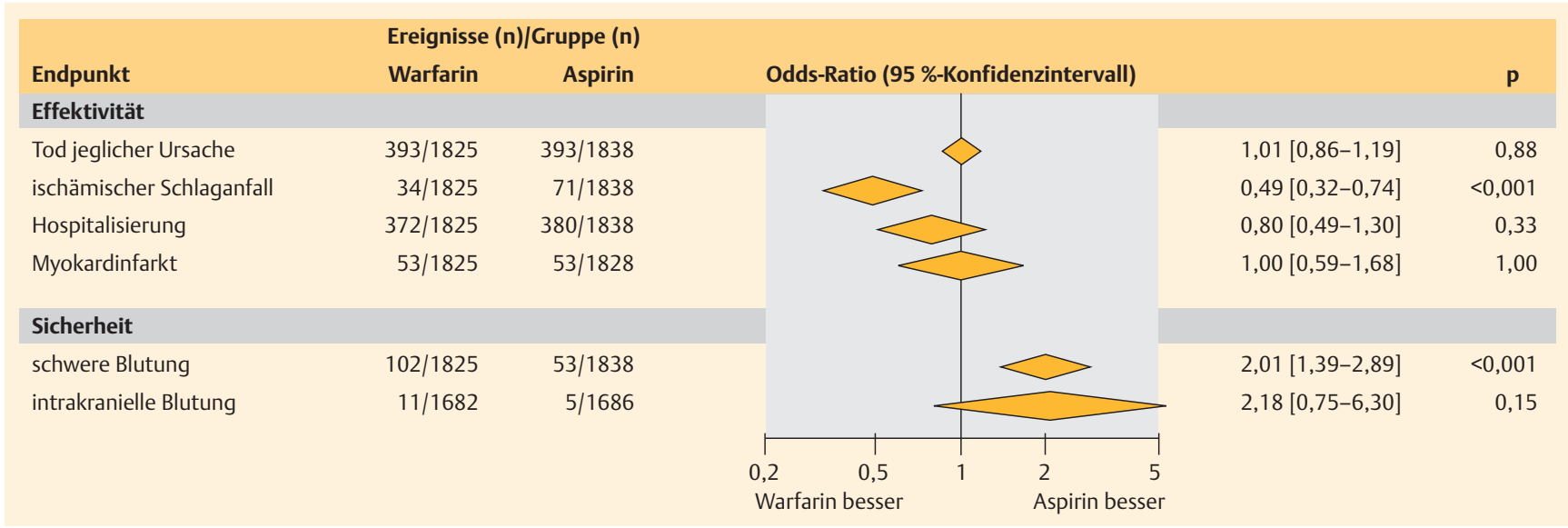

Abb. 6 Metaanalyse ASS vs. Warfarin (nach [21]).

Mortalität im Gesamtkollektiv gegenüber ASS nicht verändert. Die signifikant niedrigere Rate ischämischer Schlaganfälle unter Warfarin - die die eingangs aufgestellte Hypothese, dass eine bei Herzinsuffizienz ungünstig veränderte Virchow-Trias zu mehr thromboembolischen Ereignissen führt, unterstützt-steht einer erhöhten Rate von Blutungen gegenüber, wobei die Zahl der intrakraniellen Blutungen zwar erhöht, aber nominell niedrig ist (Abb.6). Obwohl auf den gleichen Datensätzen basierend, sind die Schlussfolgerungen der Arbeiten unterschiedlich: Während Rengo und Mitarbeiter folgern, dass kein Nettonutzen der plasmatischen Antikoagulation für die Patienten mit Sinusrhythmus und Herzinsuffizienz nachweisbar ist [21], sehen Hopper und Kollegen aufgrund der niedrigeren ischämischen Schlaganfallhäufigkeit bei parallel nur wenigen intrakraniellen Blutungen den Einsatz der plasmatischen Antikoagulation als gerechtfertigt an [22].

Die Frage, ob die Therapie mit ASS zu vermehrten kardialen Dekompensationen führt, wie in den kleineren Studien suggeriert, bleibt letztlich offen. In den genannten Metaanalysen gibt es hier kein eindeutiges Signal. Zu bedenken ist, dass die oben diskutierten Studien mit der in den USA üblichen ASS-Dosierung von $325 \mathrm{mg} / \mathrm{d}$ durchgeführt wurden. Diese Dosis kann grundsätzlich in vitro die günstigen Effekte der ACEHemmer auf den Prostaglandinstoffwechsel abschwächen, was die gesehenen Effekte erklären könnte. Aus diesen Gründen erscheint die in Europa übliche, niedrigere ASS-Dosis von $100 \mathrm{mg} / \mathrm{d}$ sinnvoller, falls ASS wegen vaskulärer Erkrankungen gegeben werden muss. Dieser Erklärungsansatz bleibt aber letztlich spekulativ.
Die aktuelle Datenlage zeigt keinen Nutzen für die generelle plasmatische Antikoagulation von Patienten mit Sinusrhythmus und eingeschränkter LV-Funktion.

\section{Ist die Gabe von ASS sinnvoll?}

Die Frage, ob die Gabe von ASS bei Sinusrhythmus und höhergradig eingeschränkter LV-Funktion sinnvoll ist, kann nur aus Subgruppen von nicht zu diesem Zweck entworfenen Studien extrapoliert werden. Eine Übersicht dieser Arbeiten legt zumindest nahe, dass kein überzeugender Nutzen für die ASS-Therapie aus den spärlichen Daten ableitbar ist [23]. Übliche Praxis, die allenfalls Expertenmeinung widerspiegelt, ist, Patienten mit vaskulärer Grunderkrankung mit ASS zu behandeln, während bei Patienten ohne KHK ASS verzichtbar ist. Dies scheint umso wichtiger bei der hohen Zahl von Tabletten, die Patienten mit fortgeschrittener Herzinsuffizienz üblicherweise einnehmen müssen, und der in Studien gezeigten suboptimalen Medikamentencompliance dieser Patienten [2].

Wenn keine KHK vorliegt, ist ASS verzichtbar.

\section{Antikoagulation bei intrakardialen Thromben}

Lokalisation. Intrakardiale Thromben sind typische Ursachen ischämischer Schlaganfälle und systemischer Embolien. Daher ist unbestritten, dass beim Nachweis eines intrakardialen Thrombus eine Antikoagulation 

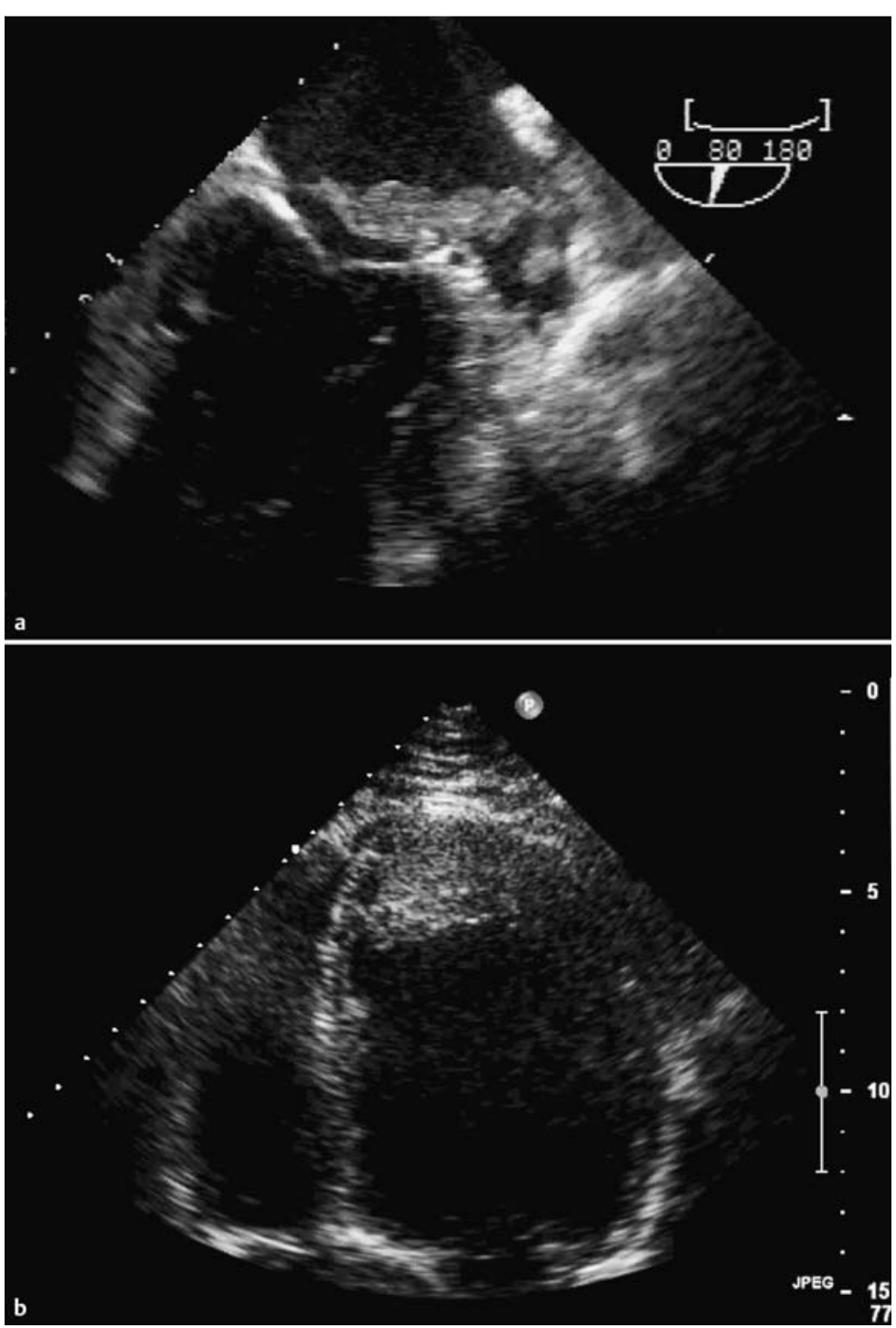

Abb. 7 Echokardiografische Darstellung intrakardialer Thromben. a Linksatrialer Thrombus bei Vorhofflimmern in der transösophagealen Echokardiografie. b Linksventrikulärer Thrombus in der transthorakalen Echokardiografie.

indiziert ist. Dabei sind 2 grundsätzliche Situationen zu unterscheiden:

- Linksatriale Thromben bei Vorhofflimmern (Abb.7a) werden nach den Leitlinien für Vorhofflimmern (s.o.) behandelt. NOAKs sind hierzu grundsätzlich zugelassen (der Zulassungstext lautet: Prophylaxe von Schlaganfällen und systemischen Embolien bei erwachsenen Patienten mit nicht valvulärem Vorhofflimmern und einem oder mehreren Risikofaktoren, was unabhängig vom Vorliegen intrakardialer Thromben ist). Die Dauer der Antikoagulation richtet sich entsprechend nach dem individuellen Risiko und wird in der Regel lebenslang durchgeführt. Ob die NOAKs eher als Warfarine zur Auflösung intrakardia- ler Thromben führen können, ist bisher nicht beantwortet.

- Linksventrikuläre Thromben (Abb. 7b) bilden sich typischerweise im linksventrikulären Apex oder in Aneurysmen/Divertikeln des linken Ventrikels. Der Nachweis oder Ausschluss linksventrikulärer apikaler Thromben kann echokardiografisch aufgrund typischerweise auftretender Nahfeldartefakte in der Echokardiographie ausgesprochen schwierig sein. Die Gabe linksherzgängiger Kontrastmittel ist ggf. sinnvoll. Eine alternative Methode zum Nachweis ist das Cardio MRT oder CT.

Therapie. Zur Therapie dieser Thromben sind aktuell lediglich Kumarine zugelassen (Zulassungstext: Behandlung und Prophylaxe von Thrombose und Embolie - ohne Einschränkungen), ohne dass es hierzu eine Evidenzbasierung gibt. Grundsätzlich wird angenommen, dass die NOAKs in dieser klinischen Situation genauso wirksam sind wie Kumarine. Ihr Einsatz ist jedoch off-label und damit aufklärungspflichtig.

Dauer der Antikoagulation. Unklar bei linksventrikulären Thromben ist, wie lange die Antikoagulation weitergeführt werden sollte. Intuitiv unstrittig ist sicher, dass Patienten, bei denen in weiteren echokardiografischen Untersuchungen Thromben im linken Ventrikel nachgewiesen werden, weiterhin antikoaguliert bleiben bzw. sogar intensiver antikoaguliert werden sollten (empirisch kann die Zugabe von ASS oder eine höhere INR diskutiert werden; Daten hierzu liegen nicht vor). Ob jedoch die Antikoagulation abgesetzt werden kann, wenn Thromben nicht mehr nachweisbar sind, ist offen. Die Leitlinine der „Heart Failure Society of America“ empfehlen eine Antikoagulation für 3 Monate nach Myokardinfarkt und Ventrikelthrombus, sonst bei Patienten mit stattgehabter Thromboembolie. In allen anderen Fällen soll die Antikoagulationsindikation von der Morphologie des Thrombus abhängig gemacht werden [2]. Die ESC und die AHA geben hier keine eindeutigen Empfehlungen.

In den Augen des Autors sollte eine unkompliziert führbare Antikoagulation in den Fällen, in denen das thrombogene Substrat persistiert (z. B. höhergradig eingeschränkte LV-Funktion, Aneurysmen) unbegrenzt weitergeführt werden. In Fällen einer klinischen Besserung (z.B. Tachymyopathie oder Myokarditis mit wieder normalisierter LV-Funktion) kann man die Antikoagulation versuchsweise beenden, wenn echokardiografisch kein Thrombus mehr nachweisbar ist. 


\section{Sonderfall: Non-Compaction- Kardiomyopathie}

Krankheitsbild. Die linksventrikuläre Non-Compaction-Kardiomyopathie, syn. „spongy“, schwammartiges Myokard (NCCM), hat in den letzten Jahren vermehrte Aufmerksamkeit erfahren. Sie geht mit ausgedünnten Ventrikelwänden mit schwammartigen Septen einher, die typischerweise das linksventrikuläre Kavum bis zur Hälfte ausfüllen. Das Bild gleicht dem embryologischen Phänotyp des Herzens in der 6. - 8. Embryonalwoche. Es wird diskutiert, dass die NCCM einer fehlenden Verdichtung des Myokards entspricht, die üblicherweise zu Beginn der Fetalperiode abgeschlossen ist. Die genaue Pathogenese ist jedoch genauso umstritten wie klare Diagnosekriterien. Erschwerend kommt hinzu, dass sich phänotypisch ein komplettes Spektrum von eindeutigen Fällen mit schwerster Trabekularisierung und hochgradig eingeschränkter Ventrikelfunktion bis zu fast normaler Morphologie darstellt. Für weitere Details sei auf aktuelle Übersichtsarbeiten verwiesen [24,25].

Antikoagulation. Die Frage, ob Patienten mit NCCM antikoaguliert werden müssen, ergibt sich intuitiv aus dem echokardiografischen Bild tiefer ventrikulärer Septen mit sehr niedrigem intrakavitärem Fluss, der zur Thrombenbildung prädisponiert. Die Datenlage ist jedoch spärlich und umfasst im Wesentlichen heterogen behandelte Patientenserien ohne kontrollierte Intervention. In Abwesenheit von Leitinienempfehlungen oder kontrollierter Studien besteht weitgehender Konsens, dass Patienten mit dem Bild einer NCCM und eingeschränkter linksventrikulärer Funktion ( $\mathrm{EF}<40 \%$ ), sichtbaren Thromben, einer Anamnese von Vorhofflimmern oder mit stattgehabten Thomboembolien antikoaguliert werden sollten. Bei normaler LV-Funktion wird eine Antikoagulation von den meisten Autoren nicht empfohlen.

\section{Was sagen die Leitlinien zur Antikoagulation bei Herzinsuffizienz}

Drei aktuelle Leitlinien nehmen Stellung zur Antikoagulation von Herzinsuffizienzpatienten unabhängig von Vorhofflimmern:

ESC. Die Europäische kardiologische Gesellschaft (ESC) legt in ihren Herzinsuffizienzleitlinien 2012 fest, dass nur Patienten mit Vorhofflimmern antikoaguliert wer- den müssen. Die Stellungnahme aus der Vorversion von 2008, dass Patienten mit intrakardialen Thromben antikoaguliert werden sollen, findet sich nicht mehr.

ACC/AHA. Die Amerikanischen Kardiologengesellschaften (ACC/AHA) definieren eine harte Indikation zur Antikoagulation bei stattgehabtem thromboembolischem Ereignis. Eine weichere Indikation besteht bei Patienten mit Prädisposition zu thromboembolischem Ereignis (Amyloidkardiomyopathie, Non-CompactionKardiomyopathie, familiäre Kardiomyopathie mit Thromboembolien bei nahen Verwandten).

HFSA. Die amerikanische Herzinsuffizienzgesellschaft (HFSA) hat 2010 (also vor Publikation der WATCH- und WARCEF-Studien) eine Antikoagulation von Patienten mit eingeschränkter EF und nach pulmonalen und systemischen Embolien empfohlen. Weiterhin gibt es hier die oben erwähnten Empfehlungen zum Umgang mit Ventrikelthromben.

Die ausführlichste Stellungnahme findet sich in einem unlängst publizierten Konsensuspapier zur Behandlung von Patienten mit Sinusrhythmus und eingeschränkter LV-Funktion [2], siehe Kasten „Kernaussagen“.

\begin{abstract}
Heart failure is a complex of symptoms caused by various etiologies, basically characterized by reduced blood flow, changes in blood composition, and the activation of the endothelium. This leads to a prothrombotic state in many patients. Despite this clearcut pathophysiology, the evidence for anticoagulation treatment of patients with heart failure is limited. Patients with heart failure and any form of atrial fibrillation have clearly to be treated with plasmatic anticoagulants. However, in patients with sinus rhythm, plasmatic anticoagulants were not more effective than aspirin in terms of survival. Thus, the decision whether to anticoagulate patients with heart failure should be made depending on the individual risk-to-benefit-ratio.
\end{abstract}

Interessenkonflikt: Vortragstätigkeit und Reisekostenunterstützung durch Boehringer Ingelheim, Bayer Vital, Pfizer und BMS. 


\section{Kernaussagen}

- Thromboembolische Komplikationen tragen zu Morbidität und Mortalität von Herzinsuffizienzpatienten bei.

- Bei Herzinsuffizienzpatienten sollte aktiv nach Indikationen für die Antikoagulation, vor allem nach paroxysmalem Vorhofflimmern, gesucht werden. Eine Risikoabschätzung für die Antikoagulation ist mit dem $\mathrm{CHA}_{2} \mathrm{DS}_{2}$ VASc- und dem HASBLED-Score möglich [3].

- Wenn eine plasmatische Antikoagulation durchgeführt wird, ist eine parallele Gabe von
Thrombozytenaggregationshemmern nicht sinnvoll (außer 12 Monate nach Myokardinfarkt).

- Wenn keine spezifische Indikation (KHK etc.) besteht, ist ASS bei Herzinsuffizienz nicht indiziert.

- Aktuell gibt es keine gute Datenlage dafür, alle Herzinsuffizienzpatienten mit plasmatischer Antikoagulation zu behandeln. Individuelle Antikoagulationsentscheidungen sind nach Abwägung von Thromboembolierisiko und Blutungsrisiko gemeinsam mit dem Patienten zu treffen.

\section{Über den Autor}

\section{Gregor Simonis}

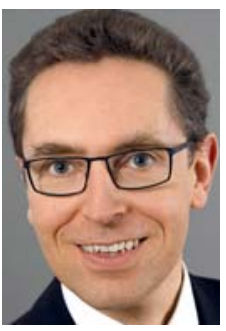

Jahrgang 1968, Prof. Dr. med. Medizinstudium in Heidelberg, klinische Ausbildung in Heidelberg und Dresden, zuletzt leitender Oberarzt in der Kardiologie des Herzzentrums Dresden Universitätsklinik. Seit 1.1.2014 Gesellschafter der Praxisklinik Herz und Gefäße Dresden.

\section{Korrespondenzadresse}

Prof. Dr. Gregor Simonis

PRAXISKLINIK Herz und Gefäße

Kardiologie - Angiologie - Radiologie - Nuklearmedizin

Akademische Lehrpraxisklinik der TU Dresden

Forststraße 3

01099 Dresden

E-Mail: Prof.Simonis@Praxis-Klinik-Dresden.de

\section{Literatur}

1 Freudenberger RS, Schumaecker MM, Homma S. What is the appropriate approach to prevention of thromboembolism in heart failure? Thromb Haemost 2010; 103: 489-495

2 Lip GYH, Piotrponikowski P, Andreotti F et al. Thromboembolism and antithrombotic therapy for heart failure in sinus rhythm. An Executive Summary of a joint Consensus Document from the ESC Heart Failure Association and the ESC

Working Group on Thrombosis. Thrombosis and Haemostasis 2012; 108: 1009 - 1022

3 Task FM et al. Developed with the special contribution of the European Heart Rhythm Association (EHRA), Endorsed by the European Association for Cardio-Thoracic Surgery (EACTS) Guidelines for the management of atrial fibrillation: The Task Force for the Management of Atrial Fibrillation of the European Society of Cardiology (ESC). Eur Heart J 2010; 31: 2369 2429

4 Task FM, Camm AJ, Lip GYH et al. 2012 focused update of the ESC Guidelines for the management of atrial fibrillation: An update of the 2010 ESC Guidelines for the management of atrial fibrillationDeveloped with the special contribution of the European Heart Rhythm Association. Eur Heart J 2012; 33 : 2719- 2747

5 Gage BF, Waterman AD, Shannon W et al. Validation of clinical classification schemes for predicting stroke: Results from the national registry of atrial fibrillation. JAMA 2001; 285: 2864 2870

6 Hohnloser SH, Pajitnev D, Pogue J et al. Incidence of Stroke in Paroxysmal Versus Sustained Atrial Fibrillation in Patients Taking Oral Anticoagulation or Combined Antiplatelet Therapy: An ACTIVE W Substudy. JACC 2007; 50: 2156-2161

7 Connolly SJ, Ezekowitz MD, Yusuf S et al. Dabigatran versus Warfarin in Patients with Atrial Fibrillation. N Engl J Med 2009; 361: 1139 - 1151

8 Patel MR, Mahaffey KW, Garg J et al. Rivaroxaban versus Warfarin in Nonvalvular Atrial Fibrillation. N Engl J Med 2011; 365: $883-891$

9 Granger CB, Alexander JH, McMurray JJV et al. Apixaban versus Warfarin in Patients with Atrial Fibrillation. N Engl J Med 2011; 365: $981-992$

10 Harper P, Young L, Merriman E. Bleeding Risk with Dabigatran in the Frail Elderly. N Engl J Med 2012; 366: 864-866

11 Boehringer Ingelheim Pharma GmbH \& Co. KG. Rote-HandBrief zu Pradaxa (Dabigatranexelitat): Notwendigkeit der Überprüfung der Nierenfunktion. 2011

12 Wolf PA, Dawber TR, Thomas HE et al. Epidemiologic assesment of chronic atrial fibrillation and the risk of stroke: the Framingham Study. Neurology 1978; 28: 973 - 977

13 Eikelboom JW, Connolly SJ, Brueckmann M et al. Dabigatran versus Warfarin in Patients with Mechanical Heart Valves. N Engl J Med 2013; 369: 1206 - 1214

14 Lip GYH, Gibbs CR. Does heart failure confer a hypercoagulable state? Virchow's triad revisited JACC 1999; 33: 1424 1426

15 Uretsky BF, Thygesen K, Armstrong PW et al. Acute Coronary Findings at Autopsy in Heart Failure Patients With Sudden Death: Results From the Assessment of Treatment With Lisinopril and Survival (ATLAS) Trial. Circ 2000; 102: 611-616

16 Cokkinos DV, Haralabopoulos GC, Kostis JB et al. Efficacy of antithrombotic therapy in chronic heart failure: The HELAS study. Eur J of Heart Failure 2006; 8: 428-432

17 Cleland JGF, Findlay I, Jafri S et al. The Warfarin/Aspirin study in heart failure (WASH): a randomized trial comparing antithrombotic strategies for patients with heart failure. Am Heart J 2004; 148: $157-164$ 
18 Massie BM, Collins JF, Ammon SE et al. Randomized Trial of Warfarin, Aspirin, and Clopidogrel in Patients With Chronic Heart Failure: The Warfarin and Antiplatelet Therapy in Chronic Heart Failure (WATCH) Trial. Circ 2009; 119: 1616-1624

19 Homma S, Thompson JLP, Pullicino PM et al. Warfarin and Aspirin in Patients with Heart Failure and Sinus Rhythm. N Engl J Med 2012; 366: 1859-1869

20 Homma S, Thompson JLP, Sanford AR et al. Benefit of Warfarin Compared With Aspirin in Patients With Heart Failure in Sinus Rhythm: A Subgroup Analysis of WARCEF, a Randomized Controlled Trial. Circulation: Heart Failure 2013; 6: 988-997

21 Rengo G, Pagano G, Squizzato A et al. Oral Anticoagulation Therapy in Heart Failure Patients in Sinus Rhythm: A Systematic Review and Meta-Analysis. PLoS ONE 2013; 8: e52952
22 Hopper I, Skiba M, Krum H. Updated meta-analysis on antithrombotic therapy in patients with heart failure and sinus rhythm. Eur J of Heart Failure 2013; 15: 69-78

23 Lip GYH, Gibbs CR. Antiplatelet agents versus control or anticoagulation for heart failure in sinus rhythm: a Cochrane systematic review. QJM 2002; 95: 461 - 468

24 Oechslin E, Jenni R. Left ventricular non-compaction revisited: a distinct phenotype with genetic heterogeneity? Eur Heart J 2011; $32: 1446$ - 1456

25 Thavendiranathan P, Dahiya A, Phelan D et al. Isolated left ventricular non-compaction controversies in diagnostic criteria, adverse outcomes and management. Heart 2013; 99: $681-689$ 


\section{CME。thieme.de}

\section{CME-Fragen}

\section{CME-Teilnahme}

- Viel Erfolg bei Ihrer CME-Teilnahme unter http://cme.thieme.de

- Diese Fortbildungseinheit ist 12 Monate online für eine CME-Teilnahme verfügbar.

- Sollten Sie Fragen zur Online-Teilnahme haben, unter http://cme.thieme.de/hilfe finden Sie eine ausführliche Anleitung.

1 Welche der folgenden Veränderungenen der Pathophysiologie treten bei Herzinsuffizienz nicht auf?

A erniedrigtes Herzzeitvolumen

B Gerinnungsaktivierung durch Stase

C endotheliale Dysfunktion

D sekundäre Thrombozytenaktivierung

E Polyglobulie

2 Welche der folgenden Aussagen trifft für Vorhofflimmern und Herzinsuffizienz zu?

A Vorhofflimmern spielt für das Thromboembolierisiko bei Herzinsuffizienz keine Rolle.

B Die neuen oralen Antikoagulanzien sind bei Herzinsuffizienz kontraindiziert.

C Nahezu alle Herzinsuffizienzpatienten mit Vorhofflimmern müssen antikoaguliert werden.

D Die Gabe von ASS ist bei Vorhofflimmern ausreichend.

E Bei Vorhofflimmern und Herzinsuffizienz sind Blutungen kein Problem.

3 Welches der folgenden Medikamente ist zur Behandlung linksventrikulärer Thromben zugelassen?
A Phenprocoumon
B ASS
C Dabigatran
D Rivaroxaban
E Apixaban

4 Welche der folgenden Aussagen ist nicht richtig?
A Neue Antikoagulanzien sind bei valvulärem Vorhofflimmern kontraindiziert.
B Mitralstenosen führen zu valvulärem Vorhofflimmern.
C Patienten mit valvulärem Vorhofflimmern haben oft ein rheumatisches Fieber in der Vorgeschichte.
D Valvuläres Vorhofflimmern tritt typischerweise im sehr hohen Alter auf.
E Sekundäre Mitralinsuffizienzen führen nicht zu typischem valvulärem Vorhofflimmern.

5 Welche der folgenden Aussagen ist nicht richtig?
A Primärer Endpunkt der HELAS-Studie war die Kombination aus Schlaganfall, Myokardinfarkt, peripherer Embolie, Lungenembolie, Hospitalisation aus kardialer Ursache und Tod jeglicher Ursache.
B Die WASH-Studie randomisierte 279 Patienten mit symptomatischer systolischer Herzinsuffizienz in keine Therapie, ASS oder Kumarintherapie.
C Die WASH-Studie war doppelblind randomisiert.
D In HELAS und WASH waren kardiale Dekompensationen häufiger als Thromboembolien.
E HELAS und WASH wiesen eine hohe Zahl von Todesfällen auf. 


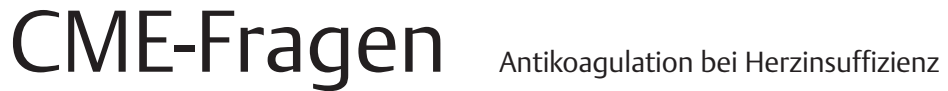

6 Welche der folgenden Aussagen ist richtig?

A Die WATCH-Studie verglich Warfarin mit Placebo.

B Die WARCEF-Studie verglich Warfarin mit Clopidogrel.

C Blutungen waren der primäre Endpunkt in der WATCH-Studie.

D WARCEF ist die größte Studie zur Antikoagulation bei Herzinsuffizienz.

E In der WATCH-Studie waren auch Patienten mit Vorhofflimmern zugelassen.

$7 \quad$ Welche der folgenden Aussagen ist nicht richtig?

A Primärer Endpunkt in WARCEF war die Kombination aus ischämischem oder hämorrhagischem Schlaganfall und der Gesamtmortalität.

B In WARCEF gab es unter Warfarin weniger Blutungen als unter ASS.

C In WARCEF profitierten vor allem jüngere Patienten von Warfarin.

D In WARCEF war der Nutzen von Falithrom nach längerer Behandlung ausgeprägter als nach kürzerer.

E Die Autoren von WARCEF folgern, dass die antithrombotische Therapie zu individualisieren ist.

8 Welche der folgenden Aussagen ist nicht richtig?

A Vorhofthromben bei Vorhofflimmern ändern das Antikoagulationsregime üblicherweise nicht.

B Neue Antikoagulanzien sind bei Vorhofthromben zugelassen.

C Intrakardiale Thromben führen zu Schlaganfällen und systemischen Embolien.

D Linksventrikuläre Thromben führen zu Lungenembolien.

E Herzwandaneurysmen prädisponieren zur Thrombenbildung.

9 Welche der folgenden Aussagen ist richtig?

A Bei eingeschränkter LV-Funktion ist die Gabe von ASS prognoseverbessernd.

B Wenn eine Antikoagulationsindikation besteht, soll die plasmatische Antikaogulation mit ASS kombiniert werden.

C Herzinsuffizienz führt zu uniformen Antikoagulationsentscheidungen.

D Die Entscheidung zur Antikoagulation sollte individuell abgewogen werden.

E Die Zahl der Tabletten, die der Patient nehmen muss, ist irrelevant.

10 Welche der folgenden Aussagen ist richtig?

A Der $\mathrm{CHA}_{2} \mathrm{DS}_{2}$ VASc-Score gilt nicht für Herzinsuffizienzpatienten.

B Der HASBLED-Score gilt nicht für Herzinsuffizienzpatienten.

C Patienten mit Herzinsuffizienz mit erhaltener LV-Funktion sind die führende Population in den Antikoagulationsstudien bei Sinusrhythmus.

D Paroxysmales Vorhofflimmern führt seltener zu Thromboembolien als permanentes Vorhofflimmern.

E Alle Patienten mit Vorhofflimmern und erhöhtem $\mathrm{CHA}_{2} \mathrm{DS}_{2}$ VASc-Score, z. B. bei Herzinsuffizienz, werden antikoaguliert unabhängig vom Typ des Vorhofflimmerns. 